BULL. AUSTRAL. MATH. SOC.

\title{
THE DIFFERENCE OPERATORS AND EXTENDED LAGUERRE POLYNOMIALS
}

\author{
B.P. PARAshar
}

A new set of polynomials $L_{n}^{(\alpha, h)}(x)$ in powers of $x^{h} \quad(h>0)$ which are related to the Laguerre polynomials $L_{n}^{(\alpha)}(x)$, have been defined by a Rodrigues type formula involving the forward difference operator $\Delta_{h}$ of increment $h$. Besides obtaining shift operator formulae for $L_{n}^{(\alpha, h)}(x)$, a definition of fractional power $\Delta_{h}^{\lambda}$ of $\Delta_{h}$ has been given. The definition has been illustrated by establishing known relations of the confluent hypergeometric function.

\section{Introduction}

The use of the forward difference operator $\Delta$ of increment 1 where

$$
\Delta f(\alpha)=f(\alpha+1)-f(\alpha)
$$

in obtaining Rodrigues type formulae for some hypergeometric type special functions is well-known. For example regarding $\alpha$ as the discrete variable, the Laguerre polynomial $L_{n}^{(\alpha)}(x)$ is given by

$$
\Delta^{n} \frac{x^{\alpha}}{\Gamma(1+\alpha)}=\frac{(-1)^{n} n ! x^{\alpha}}{\Gamma(1+\alpha+n)} L_{n}^{(\alpha)}(x)
$$

where

Received 9 May 1983. 


$$
L_{n}^{(\alpha)}(x)=\frac{(1+\alpha)}{n !}{ }_{1} F_{1}[-n ; 1+\alpha ; x]
$$

(see Erdélyi [1, p. 191]).

Further, if $\alpha$ is regarded as a continuous variable then $x^{\alpha} / \Gamma(1+\alpha)$

is an entire function of $\alpha$ and $\left(d^{n} / d \alpha^{n}\right)\left[x^{\alpha} / \Gamma(1+\alpha)\right]$ exists for all nonnegative interger values of $n$. Using this property, the $\mu$-function is defined as follows:

$$
\frac{d^{n}}{d \alpha^{n}}\left[\frac{x^{\alpha}}{\Gamma(1+\alpha)}\right]=(-1)^{n} \mu(x,+n-1, \alpha)
$$

(see Erdélyi [2, p. 218]).

Thus by the Taylor expansion,

$$
\frac{x^{\alpha+t}}{\Gamma(1+\alpha+t)}=\sum_{n=0}^{\infty} \mu(x,-n-1, \alpha) \frac{(-t)^{n}}{n !} .
$$

Also by the Gregory-Newton formula

$$
\frac{x^{\alpha+t}}{\Gamma(1+\alpha+t)}=\sum_{n=0}^{\infty}\left({ }_{n}^{t}\right) \Delta^{n} \frac{x^{\alpha}}{\Gamma(1+\alpha)},
$$

which with (1.1) gives

$$
\frac{x^{t}}{\Gamma(1+\alpha+t)}=\sum_{n=0}^{\infty} \frac{(-t) n}{\Gamma(1+\alpha+n)} L_{n}^{(\alpha)}(x),
$$

provided the infinite series is convergent.

It is our aim in this note to further generalise these results by using the operator $\Delta_{h}$ of increment $h$ given by

$$
\Delta_{h} f(\alpha)=f(\alpha+h)-f(\alpha), h>0,
$$

and also to consider its fractional power $\Delta_{h}^{\lambda}$ for arbitrary real $\lambda$.

REMARK. It may be emphasised that the novelty of the present work consists not so much in obtaining new results as in the remarkable case with which results are obtained by the difference operator procedure. 


\section{Definition of $\Delta_{h}^{\lambda}$ and its consequences}

Following the finite operator identify

(a) $\Delta_{h}^{n} \equiv \sum_{k=0}^{n}\left(\begin{array}{l}n \\ k\end{array}\right)(-1)^{k} E_{h}^{n-k}, E_{h} \equiv 1+\Delta_{h}, \Delta_{h}^{\lambda}$ is defined for arbitrary $\lambda$ as follows,

$$
\Delta_{h}^{\lambda} f(\alpha)=\sum_{k=0}^{\infty}\left(\begin{array}{l}
\lambda \\
k
\end{array}\right)(-1)^{k} f(\alpha+\lambda h-k h)
$$

provided the infinite series in (2.1) converges.

When the formula (2.1) is valid it also leads to a definition of the fractional order derivative $D^{\lambda}$ as follows:

$$
\lim _{h \rightarrow 0} \frac{\Delta_{h}^{\lambda}}{h^{\lambda}} f(\alpha)=D^{\lambda} f(\alpha)
$$

The following generalised powers will be used in the sequel:

(b) $\alpha^{(\beta, h)}=h^{\beta} \frac{\Gamma(1+(\alpha / h))}{\Gamma(1-\beta+(\alpha / h))}$;

(c) $(\alpha)_{B, h}=h^{\beta} \frac{\Gamma((\alpha / h)+\beta)}{\Gamma(\alpha / h)}$.

The use of (2.1) will then give

$$
\frac{\Delta_{h}^{\lambda}}{h^{\lambda}} \alpha^{(\beta, h)}=\frac{\sin (\pi(\alpha / h))}{\sin \pi((\alpha / h)+\lambda)} \frac{\Gamma(\lambda-\beta)}{\Gamma(-\beta)} \alpha^{(\beta-\lambda, h)}
$$

provided $\operatorname{Re}(\lambda-\beta)>0$ or $\lambda$ is a non-negative integer.

If we use the asymptotic form

$$
\frac{\Gamma(a+z)}{\Gamma(b+z)} \sim z^{a-b} \text { as }|z| \rightarrow \infty
$$

then, as $h \rightarrow 0,(2.3)$ leads to

$$
D^{\lambda} \alpha^{\beta}=(-1)^{\lambda+1} \frac{\sin \pi \beta}{\sin \pi(1+\beta-\lambda)} \frac{\Gamma(1+\beta)}{\Gamma(1+\beta-\lambda)} \alpha^{\beta-\lambda} .
$$

For $\lambda=n$ a non-negative integers, (2.4) reduces to the usual $n$th derivative of $x^{\beta}$, namely, 


$$
D^{n} \alpha^{\beta}=\frac{\Gamma(1+\beta)}{\Gamma(1+\beta-n)} \alpha^{\beta-n} .
$$

Using 2 (a) the following relations can be easily verified:

$$
\Delta_{h}^{n} \frac{x^{\alpha}}{\Gamma[1+(\alpha / h)]}=\frac{(-1)^{n} n ! x^{\alpha}}{\Gamma(1+n+(\alpha / h)]} L_{n}^{(\alpha / h)}\left(x^{h}\right),
$$

$$
\Delta_{h}^{n} \frac{x^{\alpha}}{\Gamma(1-(\alpha / h)]}=\frac{n ! x^{\alpha+n h}}{\Gamma(1-(\alpha / h))} L_{n}^{(-(\alpha / h)-n)}\left(x^{-h}\right) \text {. }
$$

Also for arbitrary $\lambda$, using (2.1) we shall have

$$
\Delta_{h}^{\lambda} \frac{x^{\alpha}}{\Gamma(1+(\alpha / h)]}=\frac{x^{\alpha+\lambda h}}{\Gamma(1+\lambda+(\alpha / h))} 2^{F_{0}}\left[-\lambda,-\lambda-(\alpha / h) ;-x^{-h}\right]
$$

which for $\lambda=n$ reduces to (2.5) because of the relation

$$
L_{n}^{(\beta)}(x)=\frac{(-x)^{n}}{n !} 2^{F_{0}}[-n,-n-\beta ;-1 / x] .
$$

Also

$$
\Delta_{h}^{\lambda} \frac{x^{\alpha}}{\Gamma(1-(\alpha / h))}=\frac{x^{\alpha+\lambda h}}{\Gamma(1-\lambda-(\alpha / h)]}{ }_{1} F_{1}\left[-\lambda ; 1-\lambda-(\alpha / h) ; x^{-h}\right] .
$$

The Leibnitz formula for $\Delta_{h}^{n}$ is given by

(d) $\Delta_{h}^{n} f g=\sum_{k=0}^{n}\left(\begin{array}{l}n \\ k\end{array}\right) \Delta_{h}^{n-k} f \cdot \Delta_{h}^{k} E_{h}^{n-k} g$ (see Jordan [3, p. 97]).

This suggests that for arbitrary $\lambda$ we have

$$
\Delta_{h}^{\lambda} f g=\sum_{k=0}^{\infty}\left(\begin{array}{l}
\lambda \\
k
\end{array}\right) \Delta_{h}^{\lambda-k} f \cdot \Delta_{h}^{k} E_{h}^{\lambda-k} g
$$

provided that the infinite series in (2.9) converges. For $\lambda=n,(2.9)$ reduces to 2 (d).

As a justification of the formula (2.9) we reestablish two relations for the confluent hypergeometric function.

EXAMPLE 1. It can be verified that

$$
\left(\Delta_{h}^{\lambda} \alpha-\alpha \Delta_{h}^{\lambda}\right) f(\alpha)=\lambda h \Delta_{h}^{\lambda-1} f(\alpha+h) \text {. }
$$

For $\lambda=n$ and $h \rightarrow 0,(2.10)$ will lead to the so-called Pincherle 
derivative of $D^{n}$ given by

$$
\left(D^{n}\right) \cdot f(\alpha)=\left(D^{n} \alpha-\alpha D^{n}\right) f(\alpha)=n D^{n-1} f(\alpha) \text {. }
$$

If we put $f(\alpha)=x^{\alpha} / \Gamma(1-(\alpha / h)) \quad$ in (2.10) and use (2.8) and (2.9) we shall have

$$
\text { (2.11) } \begin{aligned}
(\lambda h+\alpha){ }_{1} F_{1}\left[-\lambda ;-\lambda-(\alpha / h) ; x^{-h}\right]-\alpha_{1} F_{1}\left[-\lambda ; 1-\lambda-(\alpha / h) ; x^{-h}\right] \\
=\lambda h_{1} F_{1}\left[1-\lambda ; 1-\lambda-(\alpha / h) ; x^{-h}\right] .
\end{aligned}
$$

With suitable replacements (2.11) can be written in the form

(2.12) $a_{1} F_{1}[a+1 ; b+1 ; u]-b_{1} E_{1}[a ; b ; u]=(a-b){ }_{1} F_{1}[a ; b+1 ; u]$

which can be verified from the following relations of ${ }_{I} F_{1}$ (see slater [5, p. 16]) :

$$
\begin{aligned}
{ }_{1} F_{1}[a+1 ; b+1 ; u] & =\frac{b}{a} \frac{d}{d u}{ }_{1} F_{1}[a ; b ; u] ; \\
{ }_{1} F_{1}[a ; b+1 ; u] & =\frac{b}{b-a}{ }_{1} F_{1}[a ; b ; u]-\frac{b}{b-a} \frac{d}{d u}{ }_{1} F_{1}[a ; b ; u] .
\end{aligned}
$$

EXAMPLE 2. If in (2.9) we put $f=x^{\alpha} / \Gamma(1-(\alpha / h)), g=y^{\alpha}$ and use the relation (2.8) with $\Delta_{h}^{k} y^{\alpha}=y^{\alpha}\left(y^{h}-1\right)^{k}$, we shall have after proper replacements the multiplication formula (see slater [5, p. 23]), namely,

$$
{ }_{1} F_{1}[a ; b ; x y]=\sum_{n=0}^{\infty} \frac{(a) n^{x^{n}(y-1)^{n}}}{n !(b)}{ }_{1} E_{1}[a+n ; b+n ; x] \text {. }
$$

\section{The extended Laguerre polynomials}

The extended Laguerre polynomials $L_{n}^{(\alpha, h)}(x)$ in powers of $x^{h}$ $(h>0)$ are defined by the Rodrigues type formula

$$
L_{n}^{(\alpha, h)}(x)=\frac{(-1)^{n} \Gamma(1+\alpha+n h)}{n !} x^{-\alpha} \Delta_{h}^{n}\left[x^{\alpha} / \Gamma(1+\alpha)\right] .
$$

Thus 


$$
L_{n}^{(\alpha, h)}(x)=\frac{\Gamma(1+\alpha+n h)}{n !} \sum_{k=0}^{n} \frac{(-n) k^{x^{k h}}}{k ! \Gamma(1+\alpha+k h)}
$$

With this definition, (1.5) will be generalised to

$$
\frac{x^{t}}{\Gamma(1+\alpha+t)}=\sum_{n=0}^{\infty} \frac{(-t / h)}{\Gamma(1+\alpha+n h)} L_{n}^{(\alpha, h)}(x) \text {. }
$$

In the case $h=\nu$ a natural number, $L_{n}^{(\alpha, \nu)}(x)$ was denoted by Konhauser [4] by $z_{n}^{\alpha}(x, v)$ and it can be expressed as a ${ }_{l} F_{\nu}$.

For $h=1, L_{n}^{(\alpha, h)}(x)$ reduces to the Laguerre polynomial $L_{n}^{(\alpha)}(x)$ and (3.1) reduces to (1.1).

As $h+0$ using (1.2) and (3.1) we shall have

$$
\mu(x,-n-1, \alpha)=\lim _{h \rightarrow 0}\left[\frac{n ! x^{\alpha} h^{-n}}{\Gamma(1+\alpha+n h)} L_{n}^{(\alpha, h)}(x)\right] .
$$

Further if we use the relation

$$
\Delta_{h}^{n} f(\alpha)=n ! \sum_{k=n}^{\infty} \frac{h^{k}}{k !} \sigma_{k}^{n} \frac{d^{k}}{d \alpha^{k}} f(\alpha)
$$

where $\sigma_{k}^{n}$ are the stirling numbers of the second kind given by

$$
\sigma_{k}^{n}=\left[\frac{\Delta^{n} x^{k}}{n !}\right]_{x=0}
$$

(see Jordan [3, p. 168, 190]), we shall have

(3.5) $\quad L_{n}^{(\alpha, h)}(x)=x^{-\alpha} \Gamma(1+\alpha+n h) \sum_{k=n}^{\infty} \frac{h^{k}}{k !}(-1)^{n+k} \sigma_{k}^{n} \mu(x,-k-1, \alpha)$.

As $k \geq n$ the asymptotic form of $\sigma_{k}^{n}$, namely, $n ! \sigma_{k}^{n} \sim k^{n}$ for $k \rightarrow \infty$, suggests that the series in (3.5) is convergent.

\section{An expansion of $x^{\beta h}$}

For a real number $\beta$ if $1+\alpha+\beta h>0$ then 


$$
\begin{aligned}
\frac{x^{\alpha+\beta h}}{\Gamma(1+\alpha+\beta h)} & =E_{h}^{\beta} \frac{x^{\alpha}}{\Gamma(1+\alpha)} \\
& =\left(1+\Delta_{h}\right)^{\beta} \frac{x^{\alpha}}{\Gamma(1+\alpha)} \\
& =\sum_{k=0}^{\infty}\left(\begin{array}{l}
\beta \\
k
\end{array}\right) \Delta_{h}^{k} \frac{x^{\alpha}}{\Gamma(1+\alpha)} .
\end{aligned}
$$

This gives

$$
x^{\beta h}=\Gamma(1+\alpha+\beta h) \sum_{k=0}^{\infty} \frac{(-\beta)_{k}}{\Gamma(1+\alpha+k h)} L_{k}^{(\alpha, h)}(x) .
$$

The infinite series in (4.1) is absolutely and uniformly convergent on $[-1,1]$ for $1+\alpha+\beta h>0$. In view of the final result being convergent, the infinite expansion in powers of $\Delta_{h}$ is also valid.

The following special cases of (4.1) are noteworthy:

$$
x^{n h}=\Gamma(1+\alpha+n h) \sum_{k=0}^{n} \frac{(-n) k_{k}^{(\alpha, h)}(x)}{\Gamma(1+\alpha+k h)} ;
$$

$$
x^{n}=n ! \sum_{k=0}^{n}(-1)^{k}\left(\begin{array}{l}
\alpha+n \\
n-k
\end{array}\right) L_{k}^{(\alpha)}(x)
$$

(see Tricomi $[7,8.2]$ ).

\section{A. ORTHOGONALITY}

For $i \leq n$,

$$
\begin{aligned}
\int_{0}^{\infty} x^{\alpha} e^{-x_{L}}{ }_{n}^{(\alpha, h)}(x) \cdot x^{i} d x & =\frac{(-1)^{n} \Gamma(1+\alpha+n h)}{n !} \Delta_{h}^{n} \int_{0}^{\infty} \frac{e^{-x} x^{\alpha+i}}{\Gamma(1+\alpha)} d x \\
& =\frac{(-1)^{n} \Gamma(1+\alpha+n h)}{n !} \Delta_{h}^{n}(1+\alpha)_{i} .
\end{aligned}
$$

As $\Delta_{h}^{n} \alpha^{i}=h^{n} n ! \delta_{n i}$ where $\delta_{n i}$ is the Kronecker delta, for $i \leq n$ we have

$$
\int_{0}^{\infty} x^{\alpha} e^{-x} L_{n}^{(\alpha, h)}(x) \cdot x^{i} d x=(-h)^{n} \Gamma(1+\alpha+n h) \delta_{n i}
$$

which, if $h=1$, reduces to the classical result for $L_{n}^{(\alpha)}(x)$. 
B. SHIFT OPERATOR FORMULAE

If in the relation

$$
\Delta_{h}^{n}{ }^{(n, h)} f(\alpha) \equiv\left(E_{h^{\alpha-\alpha}}\right)_{n, h} f(\alpha)
$$

we put

$$
f(\alpha)=\frac{\Gamma((\alpha / h)-n+1)}{\Gamma((\alpha / h)+1)} \frac{x^{\alpha}}{\Gamma(1+\alpha)}
$$

then we get

$$
\left(E_{h^{\alpha-\alpha}}\right)_{n, h^{f}} f(\alpha)=h^{n} \Delta_{h}^{n} \frac{x^{\alpha}}{\Gamma(1+\alpha)}
$$

If we recursively use the relation

$$
\left(E_{h} \alpha-\alpha+i h\right) f(\alpha)=\frac{x^{\alpha}}{\Gamma((\alpha / h)+1)}\left(h x^{h} E_{h}-\alpha+i h\right) \frac{\Gamma((\alpha / h)-n+1)}{\Gamma(1+\alpha)}
$$

we shall get finally

(4.5) $\quad \frac{(-h)^{n} n ! \Gamma((\alpha / h)+1)}{\Gamma(1+\alpha+n h)} L_{n}^{(\alpha, h)}(x)=\left(h x^{h} E_{h}-\alpha\right)_{n, h} \frac{\Gamma((\alpha / h)-n+1)}{\Gamma(1+\alpha)}$.

On the other hand if we take $f(\alpha)=x^{\alpha} / \Gamma((\alpha / h)+1)$, we shall get

$$
(-1)^{n} n ! L_{n}^{((\alpha / h)-n)}\left(x^{h}\right)=\left(h x^{h} E_{h}-\alpha\right)_{n, h} 1 \text {, }
$$

which for $h=1$ reduces to the following result of Toscano [6],

$$
n ! L_{n}^{(\alpha)}(x)=(-x E+\alpha+1) n^{2}
$$

\section{References}

[1] Arthur Erdelyi, Wilhelm Magnus, Fritz Oberhettinger, Francesco G.

Tricomi (edited by), Higher transcendental functions, Volume II (Based, in part, on notes left by Harry Bateman. McGraw-Hill, New York, Toronto, London, 1953). 
[2] Arthur Erdélyi, Wi helm Magnus, Fritz Oberhettinger, Francesco G.

Tricomi (edited by), Higher transcendental functions, Volume III (Based, in part, on notes left by Harry Bateman. McGraw-Hill, New York, Toronto, London, 1955).

[3] Charles Jordan, Calculus of finite differences, 2nd edition (Chelsea, New York, 1960).

[4] Joseph D.E. Konhauser, "Biorthogonal polynomials, suggested by the Laguerre polynomials", Pacific J. Math. 21 (1967), 303-314.

[5] L.J. Slater, Confluent hypergeometric functions (Cambridge University Press, Cambridge, 1960).

[6] Letterio Toscano, "Operatori differenzialie polinomi di Laguerre", Matematiche (Catania) 23 (1968), 197-223.

[7] Francesco G. Tricomi, Vorlesungen über Orthogonalreihen (Die Grundlehren der mathematischen Wissenschaften, 76. SpringerVerlag, Berlin, Gottingen, Heidelberg, 1955).

Department of Mathematics,

Rani Durgawati Vishwa Vidyalaya, Jabalpur (M.P.),

India. 